\title{
The bZIP transcription factor PsBZP32 is involved in cyst germination, oxidative stress response, and pathogenicity of Phytophthora sojae
}

Yuting Sheng ${ }^{1,2+}$, Long Lin ${ }^{1 \dagger}$, Han Chen ${ }^{1}$, Tianhuizi Pu', Xiaoyun Liu' ${ }^{1}$ Suomeng Dong ${ }^{1,3,4}$, Wenwu Ye $e^{1,3,4^{*}}$ (D) and Yuanchao Wang ${ }^{1,3,4^{*}}$

\begin{abstract}
The basic leucine zipper (bZIP) transcription factor family, one of the largest and most diverse transcription factor families in eukaryotes, is associated with the development and stress responses of many eukaryotic organisms. However, their biological functions in oomycete plant pathogens are unclear. A genome-wide analysis of bZIP transcription factors in Phytophthora sojae showed that PsBZP32, which has a unique bZIP-PAS domain structure, exhibited a high transcription level during the early stages of $P$. sojae infection. We silenced PsBZP32 in $P$. sojae and found that the transformants showed defective cyst germination and pathogenicity. The transformants could not eliminate host-derived reactive oxygen species during infection and were more sensitive to oxidative stress. Their response to oxidative stress was independent of the PSBZP32 transcription level; however, subcellular localization and phosphorylation of PsBZP32 were affected by $\mathrm{H}_{2} \mathrm{O}_{2}$ stress. These results indicate that PsBZP32 is involved in regulation of $P$. sojae cyst germination, oxidative stress response, and pathogenicity.
\end{abstract}

Keywords: Phytophthora sojae, bZIP transcription factor, Cyst germination, Oxidative stress response, Pathogenicity, Phosphorylation

\section{Background}

The Phytophthora genus includes over 120 described species, which are sometimes referred to as funguslike organisms but are actually oomycetes in the kingdom Stramenopila (Martin et al. 2014). Phytophthora species are notorious plant destroyers, causing multibillion-dollar direct economic losses to agricultural production and natural ecosystems worldwide annually (Erwin and Ribeiro 1996). For example, Phytophthora infestans is the etiologic agent of potato late blight, which caused the Irish potato famine of

\footnotetext{
* Correspondence: yeww@njau.edu.cn; wangyc@njau.edu.cn

${ }^{+}$Yuting Sheng and Long Lin contributed equally to this work

'Department of Plant Pathology, Nanjing Agricultural University, Nanjing 210095, China

Full list of author information is available at the end of the article
}

the 1840s. Phytophthora sojae mainly infects soybean, causing 'damping off' of seedlings as well as stemand root-rot of mature plants (Tyler 2007).

Transcription factors (TFs), which control the rate of transcription by binding to specific DNA sequences, play crucial roles in almost all biological processes, such as regulation and coordination of growth, development, cell cycle progression, and a series of physiological and metabolic adaptations to the environment (Karin 1990). Phytophthora species or oomycetes may have different and more complex transcriptional regulation networks. For example, according to the Fungal Transcription Factor Database (v. 1.2; http://ftfd.snu.ac.kr), the average number of TFs in Phytophthora species is 736, almost twofold that (424) in fungal species. In addition, the Myb TFs in Phytophthora species have novel diversified

(c) The Author(s). 2021 Open Access This article is licensed under a Creative Commons Attribution 4.0 International License, which permits use, sharing, adaptation, distribution and reproduction in any medium or format, as long as you give

appropriate credit to the original author(s) and the source, provide a link to the Creative Commons licence, and indicate if changes were made. The images or other third party material in this article are included in the article's Creative Commons licence, unless indicated otherwise in a credit line to the material. If material is not included in the article's Creative Commons licence and your intended use is not permitted by statutory regulation or exceeds the permitted use, you will need to obtain permission directly from the copyright holder. To view a copy of this licence, visit http://creativecommons.org/licenses/by/4.0/ 
DNA-binding domains and a developmental stagespecific expression profile (Xiang and Judelson 2010). The bZIP TF family has an expanded number of genes in oomycetes, particularly in Phytophthora species (Ye et al. 2013). Also, many members are oomycete-specific with novel bZIP motif variants (according to the two conserved amino acid sites, the bZIP basic regions changed from the N-R type to C-R, R-R, V-R, N-K, or other types), indicating novel DNA-binding specificities or interactions with different factors (Gamboa-Melendez et al. 2013; Ye et al. 2013). Several specific cis-regulatory elements were identified in Phytophthora species, and many are enriched in the promoters of pathogenicityrelated genes, including lineage-specific effectors (Seidl et al. 2012). The absence of a TATA box and presence of novel motifs in promoters indicate that the basic transcriptional regulatory elements of oomycetes are likely diverged from that of model systems (Roy et al. 2013).

To date, few studies have been conducted on the biological functions of Phytophthora TFs, and more have focused on the Myb- and bZIP-family TFs. In P. infestans, the Myb-family TF Myb2R4 is a regulator of the light regime and sporulation, and Myb2R3 is essential for sporulation (Xiang and Judelson 2014). In P. sojae, silencing of PsMYB1, which encodes a R2R3-type Myb $\mathrm{TF}$, led to defective zoosporogenesis and infection (Zhang et al. 2012). Thirty-eight bZIP-encoding genes were identified in the $P$. infestans genome (GamboaMelendez et al. 2013). Pibzp1, which interacts with the sporangium-inducing kinase Pipkz1, is essential for zoospore motility and infection (Blanco and Judelson 2005). Eight bZIP TFs with novel bZIP motif variants (Asn-toCys) have been silenced in P. infestans. None were found to be essential for growth or pathogenicity, and only one bZIP (PITG_16038) plays a key role in $\mathrm{H}_{2} \mathrm{O}_{2}$ detoxification (Gamboa-Melendez et al. 2013). We predicted that 71 candidate bZIP TFs are encoded in the genome of $P$. sojae (Ye et al. 2013); however, the biological functions of these bZIP TFs are unknown.

Among the P. sojae bZIP TFs, only PsBZP32 has a Per-ARNT-Sim (PAS) domain together with the conserved bZIP domain. The PAS domain can serve as versatile sensor detecting a range of chemical and physical stimuli and regulating the activity of functionally diverse effector domains (Moglich et al. 2009). For example, in Neurospora, the PAS domain-containing TF WC-1 acts as both a blue-light photoreceptor and a positive element in the circadian negative feedback loop under constant darkness (He et al. 2005). In this study, we investigated the function of PsBZP32 on cyst germination, pathogenicity, and oxidative stress responses in $P$. sojae. We also found that the subcellular localization and phosphorylation status of PsBZP32 were regulated when $P$. sojae was under oxidative stress.

\section{Results}

PsBZP32 is a candidate bZIP TF with an additional PAS domain

Among the 71 predicted bZIP TFs in P. sojae (Ye et al. 2013), only PsBZP32 contained both the conserved bZIP domain and an additional PAS domain. Based on cDNA sequencing, we found that PsBZP32 gene contained two introns (127 and $76 \mathrm{bp}$ ) and a $1176 \mathrm{bp}$ open reading frame (ORF) encoding a 391-amino-acid protein. Conserved homologs of PsBZP32 were identified in species of Phytophthora, downy mildew, Pythium, and Saprolegnia (Fig. 1a). PsBZP32 and its homologs shared a similar domain architecture; i.e., a bZIP and PAS domain (bZIP-PAS; Fig. 1b). Using the Pfam database (http://pfam.xfam.org), we found that the bZIP-PAS domain organization was conserved in the proteins of non-oomycetes such as brown algae and diatoms; however, it is likely specific to Stramenopila.

In terms of the two well-characterized amino acid sites in the DNA-binding region of the bZIP domain, we identified PsBZP32 as the N-R type of bZIP TF (Fig. 1b), a conventional type of bZIP TF present in diverse animals, fungi, and plants (Gamboa-Melendez et al. 2013; Ye et al. 2013). Digital gene expression profiling (Ye et al. 2011) and reverse transcription-quantitative PCR (RT-qPCR) showed that the transcription levels of PsBZP32 were higher during the early infection stages than developmental stages of $P$. sojae (Additional file 1: Figure S1a and Fig. 1c), implying that PsBZP32 contributes to pathogenicity.

\section{Silencing of PsBZP32 affected the cyst germination rate and hyphal morphology in $P$. sojae}

To evaluate the biological functions of PsBZP32, we performed gene silencing in $P$. sojae by expressing the antisense full-length sequence of PSBZP32 (Additional file 1: Figure S1b). After antibiotic resistance screening, 136 transformants were obtained for confirmation by genomic PCR and RT-qPCR. Among them, four transformants (T14, T43, T81, and T87) were finally selected. PCR analysis of genomic DNA from these four transformants indicated the presence of the exogenous construct (Additional file 1: Figure S1c). RT-qPCR demonstrated that the PSBZP32 transcription levels in these four transformants were $53-71 \%$ of those in the wild-type strain (WT) and the control strain (CK; in which the backbone vector pTOR::GFP was transformed) at the mycelial stage (Fig. 1d).

Compared with WT and CK, the PsBZP32-silenced transformants exhibited no significant differences in mycelial growth, sporangium formation, zoospore production, encystment, or oospore production, but showed a difference in the cyst germination process. At $3 \mathrm{~h}$ postencystment of zoospores, the average cyst germination 
a

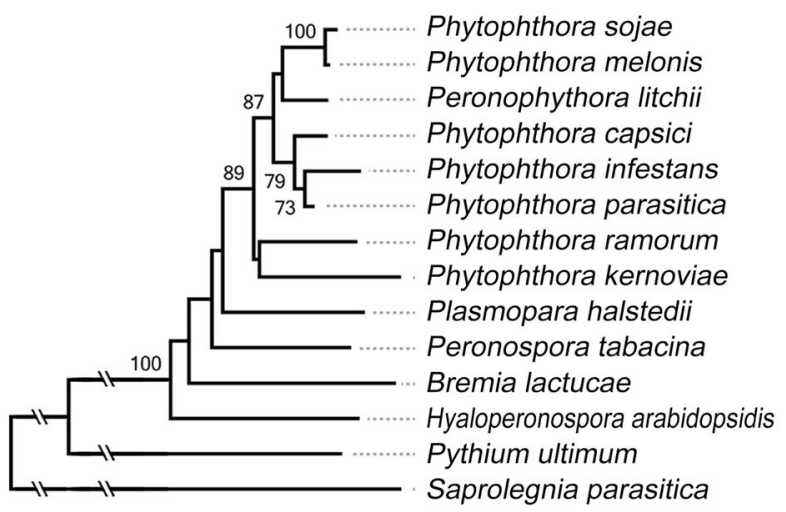

d

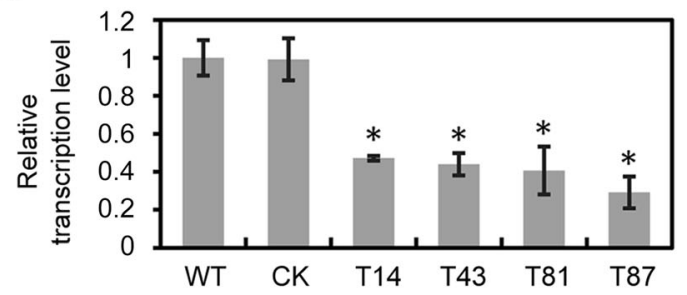

b

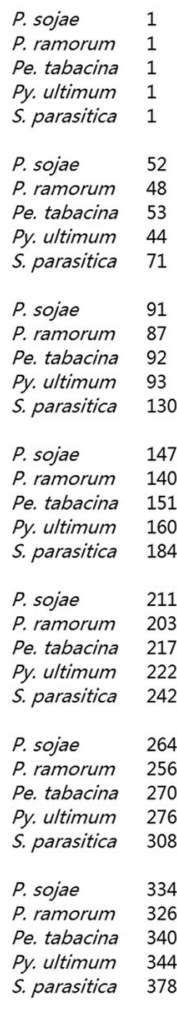

MDF T A P N E S V DMMHDD-
MDFS S P NEP D VDMMHDD-
MDF T S PNE SDVDMMHDD-

$M D F T S P S E A D V D M M Q D D$ MDNGNT F L QMDDMMDGDDLGLMDNSYNPFL TNLSNMSLAQNHHPGMHMQQRPGMHHPAMRQGQMSPAHLD 70

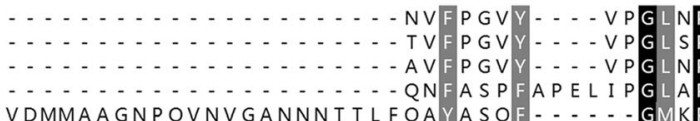

- MDQPQQAD - - . - AMEE YARLMG I KVENDD 90 - MDQPQQGD - ... AMEEYARLMG I KVENDD 86 MEQPQQGD -... AMEEYARLMG I KVENDG 91 \begin{tabular}{l} 
- GMKPATHMPDPAADAN - - SVEAYAAMMGVKLES - \\
\hline
\end{tabular}

PNV - ........ NANAFGV - PGPNNDMTKI QANAAYFENLI - SRDRNDAMDDGNNVTSMTLPAGLGS 146 PNV............. NANAFG - . P TNDMAKI QANAAYFENLI - SRDRNDAMDDGNNVTSMTLPAGIGS 139 PNVV................ A TAFGVPPGSSTDLTKIQANAAYFENLIN - TRDRTDTMDDGDNVTSMTLPAGIGG 150 DELPPSTPLAHMQAPVNAFN - L LPASDMAKI QANAAYFDDLYR - RAGSEMDLSSGANPTSMTLPAGIGS 159

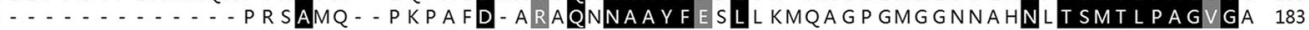

G- - MNG - FLGAPMAPAPPMYNPNTELAFQD - GGSRLYDPSSGFPGQNAPMPFHKAPQVRPGMLPPDMVG - 211

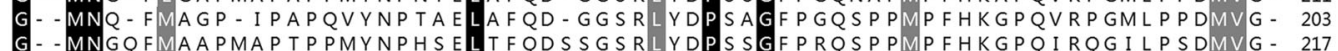
G- - MNS - FVGANSVAAAN-FGRSNDLEFGD - MNRLYNP- GAYGGGAPLQMHKPGAIGLDKFAPRIMQH 221 184 GFHMN - HVAPGHNGNSNI SNVAMPLDARAAGLVTMDGPAPGP I KAEKAMPVQ - . . . . - F S S GLVG - 242 $211 \ldots . .$. SQTLPPEI I SAMI KNN . . . . . . . . . . PAHNFENLSEDEKQALIKEEKSRERNRDHSRKSRLR 263

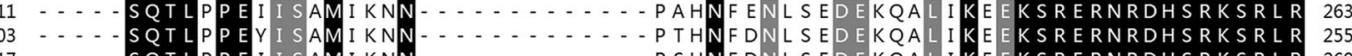

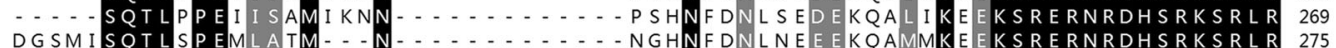
\begin{tabular}{l}
-... SQTLSPEMLSSMI S SNMQSGDVTSHSYGSNTWNASDFDYEKDALISKADKSRERNRDHSRKSRLR 307 \\
\hline
\end{tabular} KKEFVESLKHEVGQLQVYQQICEQCMDCI A LVAAEA SAVFLFSSAAYTRVLGYQNHQIVPGQTSFLDMVH 333

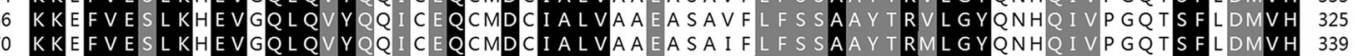

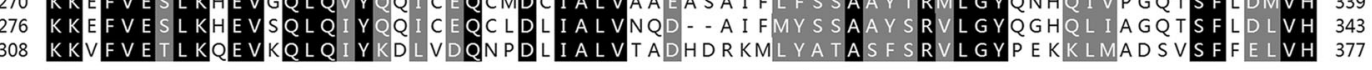

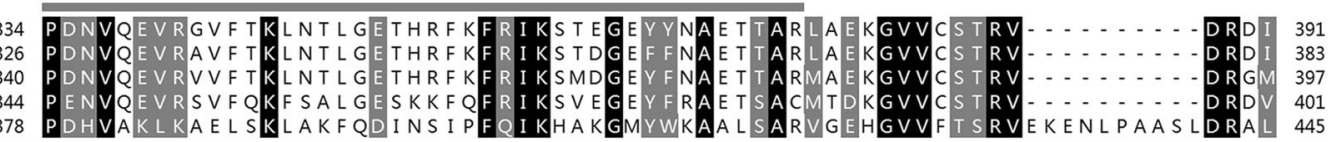

bZIP domain

PAS domain $\nabla$ conserved amino acids of bZIP domain

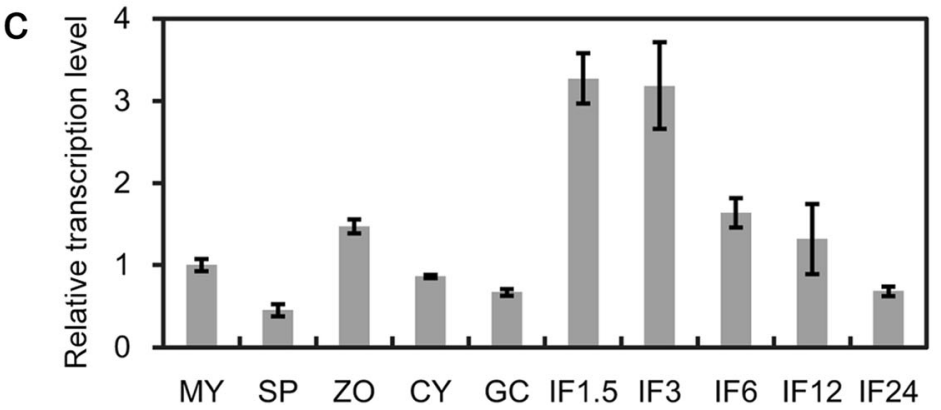

Fig. 1 (See legend on next page.) 
(See figure on previous page.)

Fig. 1 PsBZP32 is a conserved bZIP transcription factor with an additional PAS domain. a Phylogenetic analysis of PsBZP32 and its homologues in oomycetes. Full-length amino acid sequences were used to generate the phylogenetic neighbor-joining tree in MEGA 6.0. b Protein sequence alignment of PsBZP32 and its homologs in Phytophthora ramorum, Peronospora tabacina, Pythium ultimum, and Saprolegnia parasitica. c Transcription pattern of PSBZP32 according to RT-qPCR. MY, SP, ZO, CY, and GC represent the asexual life stages of $P$. sojae: vegetative mycelia, sporangia, zoospores, cysts, and germinated cysts, respectively. IF1.5, IF3, IF6, IF12, and IF24 represent the infection stages of $P$. sojae: 1.5, 3, 6, 12, and $24 \mathrm{~h}$ post-infection (hpi), respectively. Relative transcription levels were calculated using the MY values as a reference. $\mathbf{d}$ Relative transcription levels of PSBZP32 in PsBZP32-silenced transformants and control strains. WT, wild-type strain; CK, control strain transformed with empty vector. *, significant difference compared with the WT (two-tailed $t$-test, $P<0.01$ )

rate ranged from 37 to $54 \%$ in the PsBZP32-silenced transformants, significantly lower than the rates in WT (73\%) and CK (70\%) (Fig. 2a). At $10 \mathrm{~h}$ post-encystment of zoospores, significantly more abnormal hyphae (short with multiple branches) developed from the germinated cysts of the transformants (Fig. 2b), with average rates of 24-55\%, significantly higher than the rates in WT and CK (both 2\%; Fig. 2c).

\section{PsBZP32 is required for pathogenicity and invasive hyphal colonization of $P$. sojae}

Cyst germination is a key step preceding infection of $P$. sojae (Zuo et al. 2005). To explore whether PsBZP32 is involved in pathogenicity, zoospores of the PsBZP32-silenced transformants, WT, and CK were separately inoculated onto etiolated seedlings of the susceptible soybean cultivar Hefeng47. To compensate for the reduced cyst germination rate, double the zoospore number in the PsBZP32-silenced transformants compared with WT and CK were used to inoculate soybean seedlings. However, the PsBZP32-silenced transformants produced only small necrotic dark-brown spots on etiolated seedlings at $36 \mathrm{~h}$ post-infection (hpi), whereas both WT and CK produced spreading lesions (Fig. 3a).

To identify the mechanism underlying the pathogenicity defect in PsBZP32-silenced transformants, we observed zoospore-infected host tissues by microscopy and found that zoospores of WT, CK, and PsBZP32-silenced transformants penetrated epidermal cells of etiolated seedlings and produced few short infectious hyphae at 12 hpi (Fig. 3b). All strains induced reactive oxygen species (ROS) accumulation in the epidermal cells of soybean etiolated seedlings at $3 \mathrm{hpi}$. However, at $12 \mathrm{hpi}$, ROS accumulated in soybean seedlings inoculated with PsBZP32-silenced transformants, while ROS accumulation was negligible in seedlings inoculated with WT or CK (Fig. 3b), suggesting that host-derived ROS scavenging is impaired by silencing of PsBZP32 in P. sojae.

\section{PsBZP32 contributes to oxidative stress resistance, but not at the transcription level}

To determine whether PsBZP32 is involved in resistance to oxidative stress, WT, CK, and PsBZP32-silenced transformants were cultured on Plich medium supplemented with $0-5 \mathrm{mM} \mathrm{H}_{2} \mathrm{O}_{2}$. Compared with the a
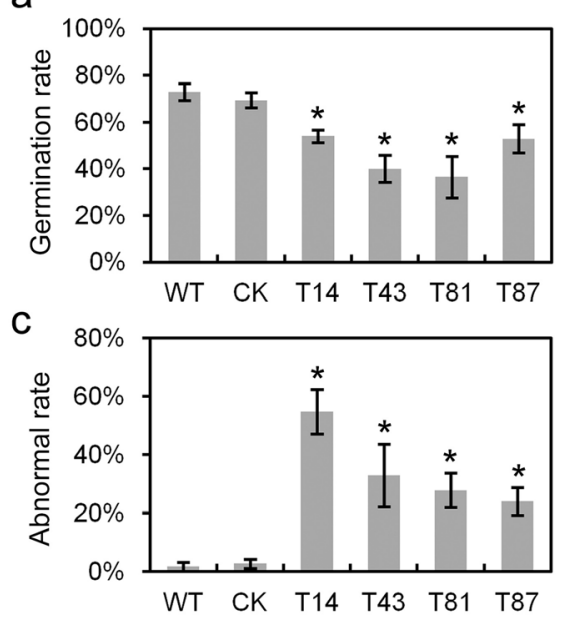

b

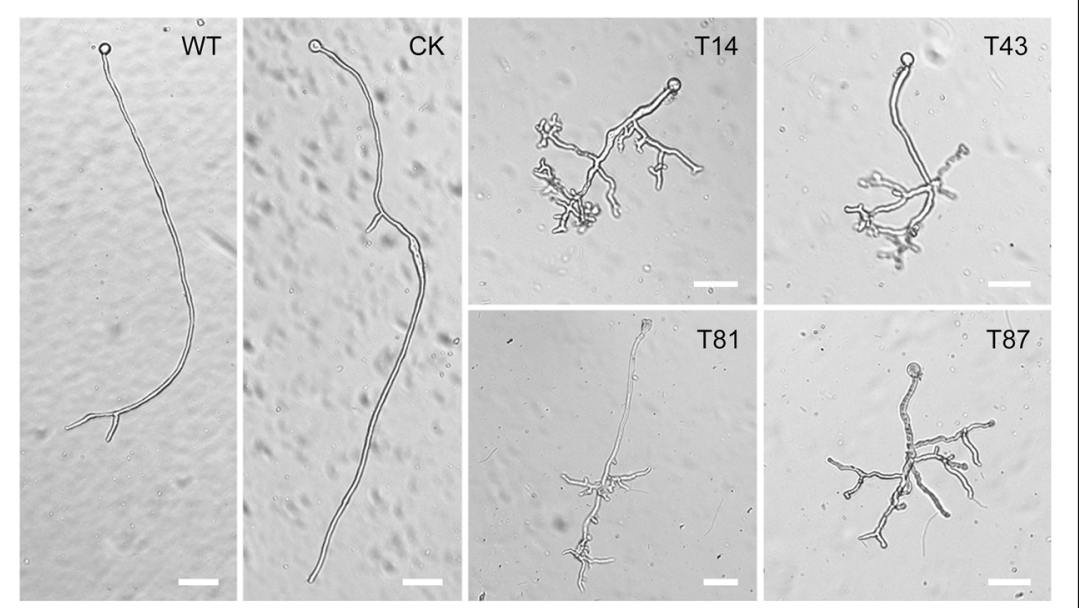

Fig. 2 Cyst germination was affected in PsBZP32-silenced transformants. a Cyst germination rate at $3 \mathrm{~h}$ after encystment. $\mathbf{b}$ Microscopic observation of germinated cysts at $10 \mathrm{~h}$ after encystment. Representative normally germinated hypha of WT and CK, and abnormally germinated hypha (short with multiple branches) of PSBZP32-silenced transformants are shown. c Abnormal rate of germinated cysts at $10 \mathrm{~h}$ after encystment. Bar, $20 \mu \mathrm{m} .{ }^{*}$, significant difference compared with the WT (two-tailed $t$-test, $P<0.01$ ) 

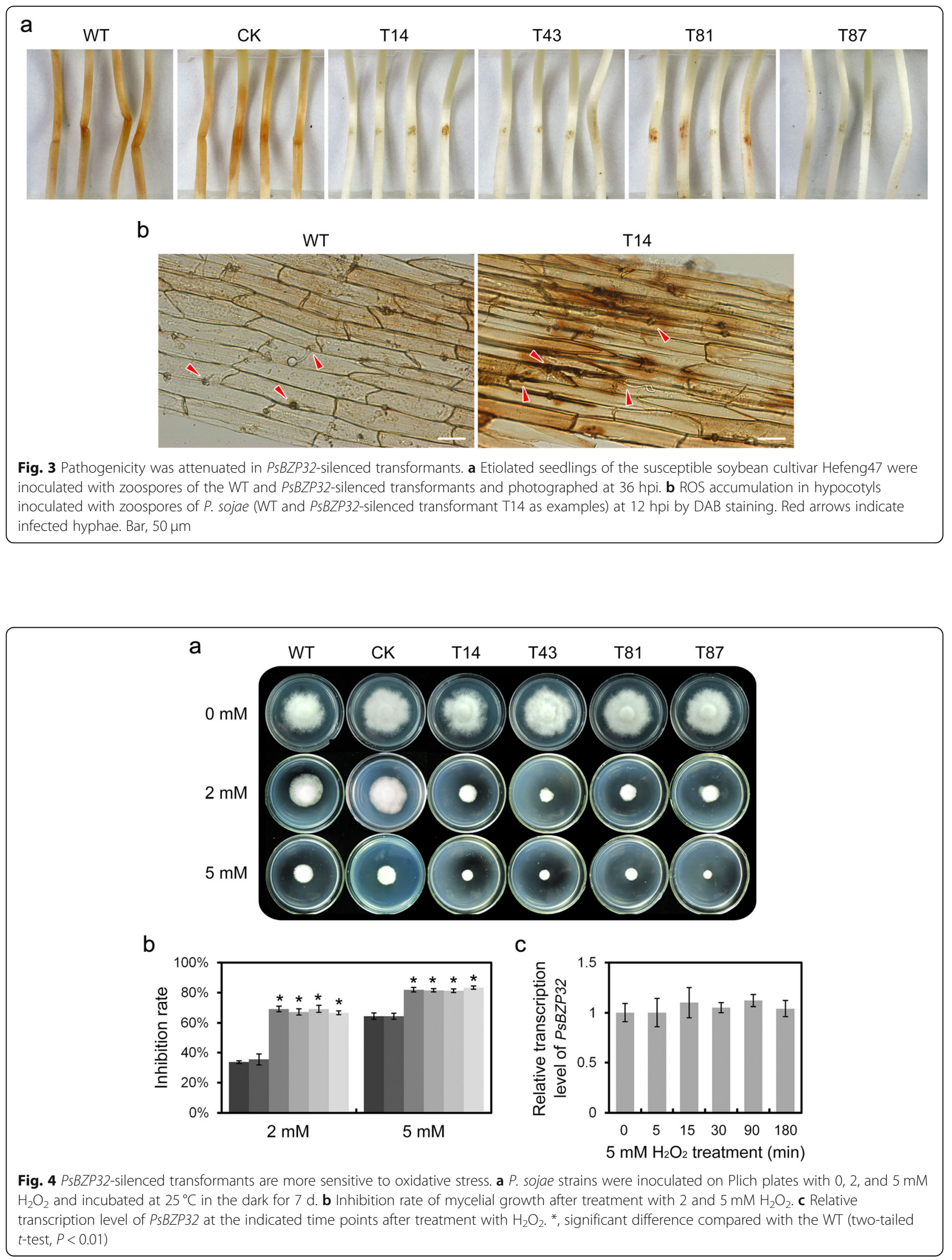
treatment without $\mathrm{H}_{2} \mathrm{O}_{2}(0 \mathrm{mM})$, all $P$. sojae strains treated with $2 \mathrm{mM} \mathrm{H}_{2} \mathrm{O}_{2}$ displayed mycelial growth inhibition, with inhibition rates of $64-68 \%$ in the PsBZP32-silenced transformants compared with 35\% and $36 \%$ in WT and CK, respectively. Similarly, when exposed to $5 \mathrm{mM} \mathrm{H}_{2} \mathrm{O}_{2}$, the PsBZP32-silenced transformants did not grow, whereas WT and CK proliferated at a low rate (Fig. 4a, b). Additionally, the transcription level of PsBZP32 in WT and CK treated with $5 \mathrm{mM}$ $\mathrm{H}_{2} \mathrm{O}_{2}$ showed no significant changes up to $180 \mathrm{~min}$ (Fig. 4c). Therefore, PsBZP32 is involved in the vegetative growth of $P$. sojae in response to oxidative stress independently of its transcription level.

The subcellular localization of PsBZP32 was modulated by oxidative stress

To assess the regulation of PsBZP32 under oxidative stress conditions, the subcellular localization of PsBZP32 was analyzed in the absence and presence of $\mathrm{H}_{2} \mathrm{O}_{2}$. A transformant was generated from the WT strain P6497, in which expression of the PsBZP32::GFP fusion protein was driven by the native promoter of PsBZP32. The GFP-expressing transformant was used as the control. In the PsBZP32::GFP-expressing strain without oxidative stress, green fluorescence was observed throughout the cells in 54\% of hyphae versus concentrated in nucleuslike spots in the remaining $46 \%$ of hyphae. After treatment with $5 \mathrm{mM} \mathrm{H}_{2} \mathrm{O}_{2}$ for $10 \mathrm{~min}$, the proportion of hyphae with green fluorescence concentrated in nucleuslike spots was increased to $86 \%$ (Fig. $5 \mathrm{a}, \mathrm{b}$ ). The green fluorescent spots were shown to be nuclei by DAPI staining (Fig. 5a). In contrast, green fluorescence was distributed throughout hyphae cells of the GFP-expressing control strain (Fig. 5a). Therefore, changes in the subcellular localization of PsBZP32 may be related to its role in the oxidative stress response.

\section{PsBZP32 was differentially phosphorylated under oxidative stress}

According to KinasePhos (http://kinasephos.mbc.nctu. edu.tw), a web server used to predict phosphorylation sites (Huang et al. 2005), nine serine residues, three threonine residues, and four tyrosine residues were predicted in PsBZP32, with a prediction specificity of 95\% (Additional file 2: Figure S2), implying that PsBZP32 is phosphorylated.

We next performed Phos-tag SDS-PAGE, normal SDSPAGE, and western blot analyses of the phosphorylated levels of PsBZP32 in the cytoplasm and nucleus of cells treated with or without $\mathrm{H}_{2} \mathrm{O}_{2}$ (Fig. $5 \mathrm{c}$, d). In the nuclear protein samples, one slower-migrating band in addition to the main band was detected by Phos-tag SDS-PAGE, compared with normal SDS-PAGE, and this band was more obvious in the $\mathrm{H}_{2} \mathrm{O}_{2}$-treated sample. The migrating band indicated that PsBZP32 might be phosphorylated. Similarly, in the cytoplasm samples, one slower-migrating band in

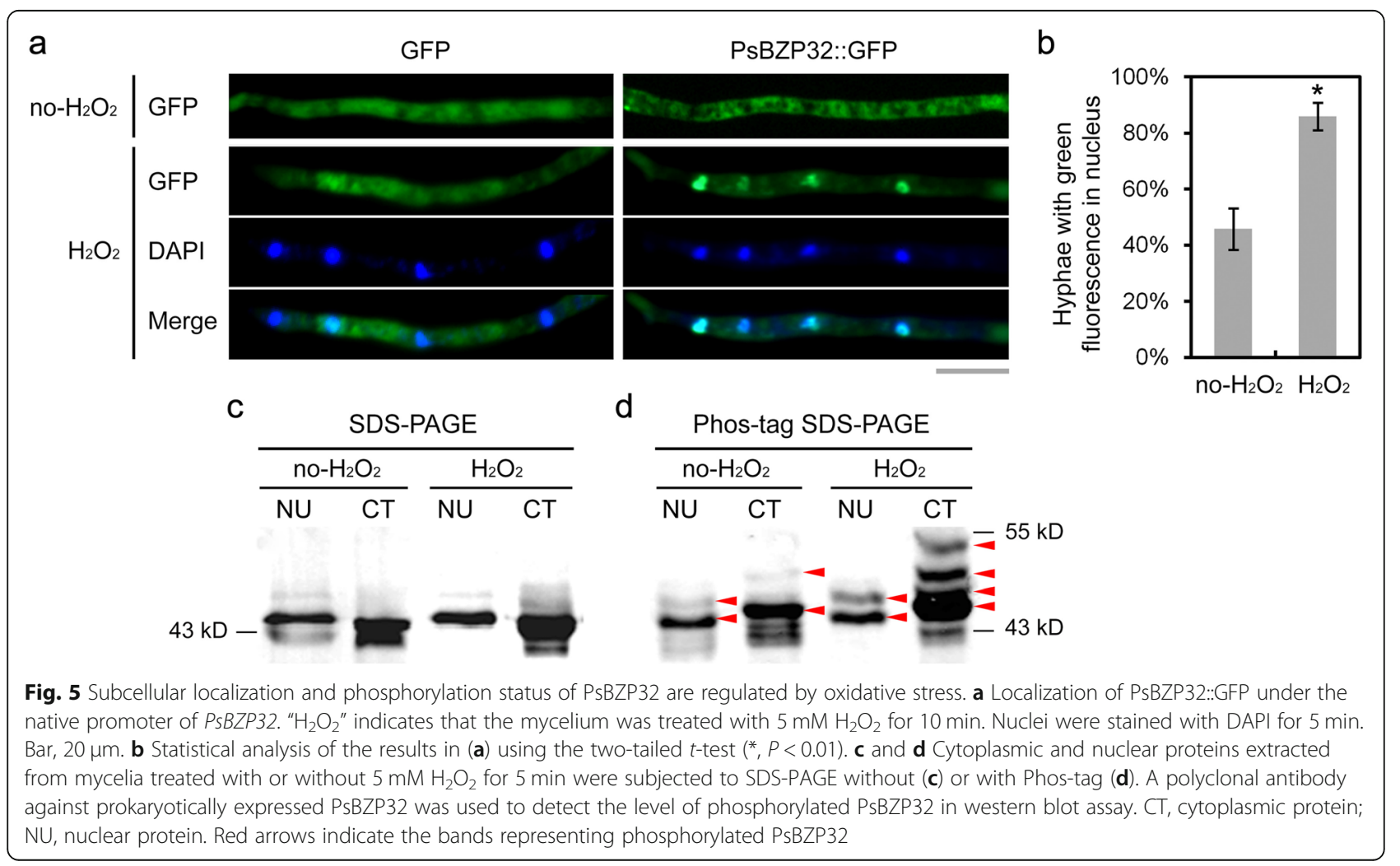


addition to the main band was observed on the Phos-tag gel in the absence of $\mathrm{H}_{2} \mathrm{O}_{2}$ treatment. However, in the presence of $\mathrm{H}_{2} \mathrm{O}_{2}$, there were two additional slower-migrating bands on the Phos-tag gel. The difference in the number and migration distance of the migrating bands indicated different phosphorylation forms and levels of the PsBZP32 protein. The phosphorylation level and pattern likely increased when the pathogen was under oxidative stress, and PsBZP32 was present in the cytoplasm.

\section{Discussion}

We reported an expanded number (71) of bZIP candidates in oomycetes, but only PsBZP32 contains an additional PAS domain (Ye et al. 2013). PAS domaincontaining proteins occur in all kingdoms of life, and this domain mediates protein-protein interactions to sense diverse signals (Moglich et al. 2009). According to our bioinformatics analysis, this bZIP-PAS protein is conserved and specific to Stramenopila. In this study and a recent study, the gene silencing assays indicated that the bZIP-PAS protein PsBZP32 plays a key role in the cyst germination, oxidative stress response, and pathogenicity of $P$. sojae, while PIBZP32 in Peronophythora litchii has similar biological functions (Kong et al. 2020). Therefore, the bZIP-PAS proteins may have conserved functions in oomycete plant pathogens.

Zoospores, the main vector for dispersal of $P$. sojae in the field, can swim chemotactically toward isoflavones released by soybean root. When a zoospore encounters the root, it transforms into an adhesive cyst, which then germinates to produce hyphae and initiate infection (Tyler 2007). Silencing of PsBZP32 reduced the cyst germination rate and resulted in abnormally germinated cysts. The abnormally germinated cysts lost polarized growth and developed into multi-branched hyphae. By contrast, PsBZP32-silenced transformants showed normal mycelial growth, sporangium formation, zoospore production, encystment, and oospore production. Therefore, PsBZP32 may regulate $P$. sojae development during cyst germination.

PsBZP32-silenced transformants showed defective pathogenicity, producing only necrotic dark brown spots at the inoculation site. They could not eliminate hostderived ROS during infection and were hypersensitive to $\mathrm{H}_{2} \mathrm{O}_{2}$. Therefore, PsBZP32 is essential for ROS detoxification at the growth and infection stages of $P$. sojae. In Pe. litchii, PIBZP32 can regulate the activities of extracellular peroxidases and laccases (Kong et al. 2020), and the heat shock TF PsHSF1 in P. sojae also has these functions (Sheng et al. 2015). However, silencing of PsBZP32 did not attenuate extracellular peroxidase and laccase activities. Therefore, PsBZP32 may regulate ROS detoxification via other pathways.
PsBZP32 showed no significant change in its transcription level under $\mathrm{H}_{2} \mathrm{O}_{2}$ stress, indicating that it regulates the oxidative stress response independently of its transcription level. The subcellular localization of bZIP is important for its transcriptional regulation (Llorca et al. 2014). MoAP1, a bZIP TF that mediates the oxidative stress response in Magnaporthe oryzae, was more concentrated in the nucleus in the presence than absence of $\mathrm{H}_{2} \mathrm{O}_{2}$ (Guo et al. 2011). Also, PsBZP32 translocated from the cytoplasm to the nucleus under $\mathrm{H}_{2} \mathrm{O}_{2}$ treatment. The subcellular localization and activity of bZIPs are regulated by protein modifications, including phosphorylation (Llorca et al. 2014). Subcellular localization of MoCRZ1, a calcineurin-responsive TF in M. oryzae, is regulated by phosphorylation. When dephosphorylated by calcineurin, it translocates from the cytoplasm to the nucleus to regulate $\mathrm{Ca}^{2+} /$ calcineurin-dependent gene expression. Next, MoCRZ1 is phosphorylated in the nucleus and translocated to the cytoplasm (Choi et al. 2009; Kim et al. 2010). We found that the phosphorylation status of PsBZP32 in the cytoplasm, but not in the nucleus, was changed by $\mathrm{H}_{2} \mathrm{O}_{2}$. However, the relationship between PsBZP32 localization and phosphorylation is unclear.

CRISPR/Cas9-mediated gene knockout in P. sojae was established in 2016 (Fang and Tyler 2016). In this study, we performed this assay at least twice in order to knockout PsBZP32 in P. sojae, although over two hundred transformants were obtained from antibiotic resistance screening, no one could be confirmed to obtain PsBZP32 knockout. In Pe. litchii, the researchers also failed to knock out PlBZP32 (Kong et al. 2020). In addition, we generated PsBZP32-overexpressing transformants, but they showed only 1.5-to-3-fold expression levels and no different phenotype compared with WT. These results indicated that expression level of BZP32 may be accurately regulated and the functions of BZP 32 may be essential for the survival or basic development of oomycetes. Considering that gene silencing might cause off-target effects, we confirmed that the transformed antisense sequence of PSBZP32 had no potential target ( $>21$ bp identical sequence) in $P$. sojae genome based on bioinformatics analysis, and in Pe. litchii, PlBZP32-silencing did not affect the expression of the neighboring gene Pl105397 (Kong et al. 2020). However, due to the technical limitations on BZP32 knockout (and complementation), the accurate biological functions of these proteins still demand further investigation.

\section{Conclusions}

We identified a bZIP-PAS type TF, PsBZP32, which exhibited a high expression level during early infection of P. sojae. Silencing of PsBZP32 led to a defect in cyst germination and pathogenicity in P. sojae. PsBZP32- 
silenced $P$. sojae transformants could not eliminate hostderived ROS during infection and were more sensitive to oxidative stress. The response of PsBZP32-silenced transformants to oxidative stress was independent of the transcription level of PsBZP32; however, the subcellular localization and phosphorylation of PsBZP32 were influenced by $\mathrm{H}_{2} \mathrm{O}_{2}$. Therefore, PsBZP32 is an important regulator of the cyst germination, oxidative stress response, and pathogenicity of $P$. sojae. Further work should focus on the mechanism underlying the change in the subcellular localization of PsBZP32 in response to oxidative stress and the function of the PAS domain in signal sensing. Also, the target genes of PsBZP32 need to be investigated to understand transcriptional regulation during oxidative stress in oomycetes.

\section{Methods}

\section{$P$. sojae growth conditions and tissue harvest}

P. sojae strain P6497 (race 2) provided by Prof. Brett Tyler (Oregon State University, USA) was used as the WT. WT and all transgenic lines in this study were routinely grown on $10 \% \mathrm{~V} 8$ medium at $25^{\circ} \mathrm{C}$ in the dark (Erwin and Ribeiro 1996). Samples from different asexual life stages (mycelia, sporangia, zoospores, cysts, germinated cysts) and infection stages ( $P$. sojae-soybean interaction for $1.5,3,6,12$, and $24 \mathrm{~h}$ ) were prepared as described ( $\mathrm{Ye}$ et al. 2011). To prepare $\mathrm{H}_{2} \mathrm{O}_{2}$-treated samples, $P$. sojae mycelia cultured in $10 \%$ V8 liquid medium at $25^{\circ} \mathrm{C}$ in the dark for $3 \mathrm{~d}$ were washed with Plich liquid medium (van West et al. 1999) and incubated in $20 \mathrm{~mL}$ Plich liquid medium supplemented with $5 \mathrm{mM} \mathrm{H}_{2} \mathrm{O}_{2}$. Mycelia were harvested after incubation for $0,5,15,30,90$, and $180 \mathrm{~min}$. Tissues were immediately frozen in liquid nitrogen followed by grinding to extract RNA for gene expression analysis by RT-qPCR as described previously (Ye et al. 2011).

\section{Vector construction and transformation in P. sojae}

The pTOR::GFP vector, provided by Prof. Franicne Govers (Wageningen University, The Netherlands), was used to generate constructs for $P$. sojae transformation. The full-length PsBZP32 ORF was amplified from cDNA of $P$. sojae strain P6497 using the primers PsBZP32XbaI-F (GCTCTAGAATGGACTTCACGGCGCCCAA) and PsBZP32-EcoRI-R (CGGAATTCTTAAATGTCG CGGTCCACGCGCGTC) and PrimeStar polymerase (Takara Bio Inc., Otsu, Japan). The amplified product was digested with $X b a \mathrm{I}$ and EcoRI, and then ligated in the antisense orientation into the digested pTOR::GFP vector. The sequenced plasmid was used for $P$. sojae transformation to generate PsBZP32-silenced transformants as described (Hua et al. 2008). The backbone vector pTOR::GFP was used to generate the control strain of $P$. sojae. The $P$. sojae transformants were screened for the PsBZP32 transgene by amplifying their genomic DNA as described (Hua et al. 2008). Total RNA was extracted from the PsBZP32-silenced transformants, and RT-qPCR was performed to analyze transcription levels.

The full-length PsBZP32 (with termination codon deleted) ORF was amplified from cDNA of P6497 using the primers PsBZP32-ClaI-F (ccATCGATATGGACTTCACGGCGCCCAA) and PsBZP32-BsiwI-R (gcCGTACGAATGTCGCGGTCCACGCGCGTC) and PrimeStar polymerase (Takara Bio Inc.). The amplified product was ligated in the sense orientation into the digested pTOR:: GFP vector. The promoter sequence of PsBZP32, $922 \mathrm{bp}$ upstream of the PSBZP32 start codon, was amplified using the primers pPsBZP32-SnabI-F (TACGTATTCA ACCCCGGCCATCGCCA) and pPsBZP32-ClaI-R (ATCGATGGCTGGGTGTGGCGTCTCCT), and the amplified product was ligated into the digested pTOR:: PsBZP32::GFP vector to replace the pHam34 promoter. The pPsBZP32::PsBZP32::GFP plasmid was sequenced and transformed into P. sojae for PsBZP32 localization analysis.

\section{Cyst germination assay}

Zoospores were induced and harvested into $1.5 \mathrm{~mL}$ tubes (Ye et al. 2013). Tubes containing at least 20 zoospores/ $\mu \mathrm{L}$ in a $500 \mu \mathrm{L}$ suspension were vortexed for $90 \mathrm{~s}$ to induce encystment. Droplets $(5 \mu \mathrm{L})$ of the cyst suspension were spotted on $0.5 \%$ V8 medium and incubated at $25^{\circ} \mathrm{C}$ for $3 \mathrm{~h}$ to induce germination. The germination of at least 100 cysts per strain was examined, and all assays were replicated three times, each in triplicate.

Zoospores in $0.5 \% \mathrm{~V} 8$ liquid medium were incubated at $25^{\circ} \mathrm{C}$ for $10 \mathrm{~h}$ following encystment. The phenotypes of 100 germinated cysts were examined using the Olympus 1X71 inverted microscope. The abnormal rate of germinated cysts was analyzed by two-tailed $t$-test $(P<0.01)$.

\section{Oxidative stress sensitivity assay}

The sensitivity of PSBZP32-silenced transformants to $\mathrm{H}_{2} \mathrm{O}_{2}$ was evaluated on modified Plich medium plates with 0 , 2, and $5 \mathrm{mM} \mathrm{H}_{2} \mathrm{O}_{2}$. Fresh $5 \mathrm{~mm}$ hyphal plugs from the WT, CK, and PsBZP32-silenced transformants were transferred from 10\% V8 plates to modified Plich medium without or with 2 or $5 \mathrm{mM} \mathrm{H}_{2} \mathrm{O}_{2}$ and incubated at $25^{\circ} \mathrm{C}$ for $7 \mathrm{~d}$. The diameter of each line was measured, and the growth rate and inhibition rate were calculated. The inhibition rate was calculated as (growth rate on plates without $\mathrm{H}_{2} \mathrm{O}_{2}$ - growth rate on plates with $\left.\mathrm{H}_{2} \mathrm{O}_{2}\right) \div$ growth rate on plates without $\mathrm{H}_{2} \mathrm{O}_{2}$. Data were subjected to statistical analysis by twotailed $t$-test $(P<0.01)$. The experiment was repeated three times, each in triplicate. 


\section{Pathogenicity assay}

Zoospores and mycelia were inoculated onto hypocotyls of etiolated soybean seedlings of cultivar Hefeng47, which is compatible with P. sojae strain P6497 ( $\mathrm{Li}$ et al. 2013), and incubated at $25^{\circ} \mathrm{C}$ in the dark for $36 \mathrm{~h}$; the result was then photographed. Zoospores and mycelia were inoculated on leaves of Hefeng 47 and incubated at $25^{\circ} \mathrm{C}$ in the dark for $3 \mathrm{~d}$; the result was then photographed. Before inoculation, hypocotyls of etiolated soybean seedlings and leaves of cultivar Hefeng47 were scratched using a pipette tip for the wound-inoculation assay. All experiments were repeated three times; each line was inoculated onto at least four seedlings or leaves.

\section{Staining}

To visualize the accumulation of $\mathrm{H}_{2} \mathrm{O}_{2}$ on hypocotyls of etiolated seedlings at $12 \mathrm{hpi}, 3$, 3-diamino benzidine (DAB; D8001, Sigma, St. Louis, MI, USA) staining was performed. Briefly, etiolated seedlings inoculated with zoospores for $12 \mathrm{~h}$ were collected, placed in $1 \mathrm{mg} / \mathrm{mL}$ $\mathrm{DAB}$ for $16 \mathrm{~h}$ in the dark at room temperature, and decolorized in ethanol (96\%) for $48 \mathrm{~h}$ (Molina and Kahmann 2007). The epidermis of DAB-stained soybean hypocotyls was visualized using the Olympus 1X71 inverted microscope.

DAPI staining was performed to visualize nuclei. Mycelia were incubated in $10 \mu \mathrm{g} / \mathrm{mL}$ DAPI for $5 \mathrm{~min}$ in the dark at room temperature, washed in sterilized distilled water twice, and observed under the Olympus 1X71 inverted microscope using the DAPI filter for blue fluorescence. To measure the localization of PsBZP32::GFP in each treatment, 100 hyphae tip (or hyphae fragments closed to tip) displaying green fluorescence anywhere were selected to calculate the proportion of those with green fluorescence in nucleus; each experiment was performed at least three times using independent samples.

\section{Nucleic acid extraction and RT-qPCR}

Genomic DNA was isolated from hyphae of P. sojae strain P6497 and all transgenic lines grown in 10\% V8 liquid medium using the Plant DNA Kit (Omega Bio-tek, USA) and subjected to PCR. Total RNA was extracted from mycelia of the WT at various developmental and infection stages. Also, mycelia were treated with $\mathrm{H}_{2} \mathrm{O}_{2}$, and RNA was extracted with the Abion Extraction Kit (Life Technology, USA), following the manufacturer's procedures, and quantified using a spectrophotometer (ND-1000; Nanodrop, USA). The total RNA integrity was confirmed by agarose gel electrophoresis.

RT-qPCR was performed to determine the transcription levels of target genes. First-strand cDNA was synthesized using M-MLV reverse transcriptase (RNase- free, Invitrogen, Shanghai, China) and oligo (dT)18 primer (Invitrogen). A $20 \mu \mathrm{L}$ reaction volume was prepared using the SYBR Prime Script RT-PCR kit (Takara Bio Inc.) according to the manufacturer's instructions. qPCR was performed on the ABI PRISM 7500 Fast Real-Time PCR System (Applied Biosystems, USA) following the manufacturer's instructions. Relative transcription levels were analyzed using 7500 System Sequence Detection Software. Primers used were designed using Primer 3.0 Input (v. 0.4.0) and commercially synthesized (Invitrogen). The primers used to amplify PsBZP32 were PsBZP32-qRT-F (CGTCTGCGCAAGAAAGAGTTC) and PsBZP32-qRT-R (GCGCTCGAGAAAAGGAAC AC). actA (PsACTA-qRT-F: ACTGCACCTTCCAGAC CATC; PsACTA-qRT-R: CCACCACCTTGATCTTCA TG) from $P$. sojae was amplified as a constitutively expressed endogenous control, and the transcription levels of target genes relative to that of actA were calculated by the $2^{-\Delta \Delta C T}$ method. All experiments were performed at least three times using independent RNA samples.

\section{Immunodetection}

Hyphae cultured in $10 \%$ V8 liquid medium at $25^{\circ} \mathrm{C}$ in the dark for $3 \mathrm{~d}$ were washed twice with SDS and transferred to SDW in the presence or absence of $5 \mathrm{mM}$ $\mathrm{H}_{2} \mathrm{O}_{2}$ for $5 \mathrm{~min}$. Hyphal tissues were immediately frozen in liquid nitrogen followed by grinding for cytoplasmic and nuclear protein extraction. Cytoplasmic protein extraction was performed using $1 \times$ PBS, $1 \%$ protease inhibitor cocktail (Sigma, P8340), 1\% phosphatase inhibitor cocktail (Sigma, P0044, P5726), and $1 \mathrm{mM}$ phenylmethylsulfonyl fluoride (ST506; Beyotime, Shanghai, China). Nuclear protein extraction was performed as described previously (Xu et al. 2012). Protein samples were added to SDS-PAGE sample loading buffer (Beyotime, P0015L), boiled for 5 min, subjected to $12 \%$ SDS-PAGE and Phos-tag SDS-PAGE, and transferred onto Immobilon-P membranes (IPVH00010; Millipore, USA) for western blotting using a rabbit polyclonal antibody specific to PsBZP32 (provided by Zoobio, Nanjing, China) and a rabbit anti-mouse IgG (926-32,211; Odyssey, Licor, USA). Phos-tag SDS-PAGE gels were prepared according to the instructions of the manufacturer of Phos-tag Acrylamide (304-93,526; Poppard, China). Anti-PsBZP32 was prepared using Escherichia coli-expressed PsBZP32 as the antigen.

\section{Bioinformatics analysis}

Protein sequences of PsBZP32 homologs in non-P. sojae species were identified by BLASTP searches (McGinnis and Madden 2004) of the genomic databases of NCBI (http://www.ncbi.nlm.nih.gov), EumicrobeDB (https:// 
www.eumicrobedb.org), and Joint Genome Institute (JGI) (http://www.jgi.doe.gov/). The obtained sequences were submitted to NCBI-CDD (http://www.ncbi.nlm.nih. gov/Structure/cdd) and SMART (http://smart.embl-heidelberg.de/) to predict conserved functional domains (Schultz et al. 1998; Letunic et al. 2015). Sequences were aligned using Clustal W (Thompson et al. 1994), and phylogenetic trees were constructed in MEGA 6.0 (Tamura et al. 2007) using neighbor-joining algorithms and 1000 bootstrap replications.

\section{Supplementary Information}

The online version contains supplementary material available at https://doi. org/10.1186/s42483-020-00078-3.

Additional file 1: Figure S1. Transcription pattern of $P_{S} B Z P 32$ and generation of PsBZP32-silenced transformants. a Transcription pattern of PsBZP32 obtained from the P. sojae Digital Gene Expression database (Ye et al. 2011). MY, SP, ZO, CY, and GC represent the asexual life stages of $P$. sojae: vegetative mycelia, sporangia, zoospores, cysts, and germinated cysts, respectively. IF1.5, IF3, IF6, IF12, and IF24 represent the infection stages of $P$. sojae: $1.5,3,6,12$, and 24 hpi, respectively. b Schematic diagram of the construct used to silence PSBZP32 in P. sojae. A reverse fragment of full-length PSBZP32 was expressed with the strong promoter pHAM34 to silence PSBZP32. tHAM34 indicates the terminator. Reversed PSBZP32 was inserted between PHAM34 and tHAM34 in the pTOR vector. c Genomic DNA PCR (using pTOR primers pTOR-F and PTOR-R indicated in $\mathbf{b}$ ). The exogenous fragment was integrated into the $P$. sojae genome.

Additional file 2: Figure S2. Phosphorylation sites predicted from the PsBZP32 protein sequence. Prediction was performed using KinasePhos (http://kinasephos.mbc.nctu.edu.tw).

\section{Abbreviations}

bZIP: Basic leucine zipper; ORF: Open reading frame; PAS: Per-ARNT-Sim; TF: Transcription factor; RT-qPCR: Reverse transcription quantitative PCR; WT: Wild-type strain; CK: Control strain transformed with empty vector; ROS: Reactive oxygen species

\section{Acknowledgements}

We are grateful to Prof. Brett Tyler at Oregon State University for suggestions and discussion.

\section{Authors' contributions}

YW, WY, and SD conceived and designed the experiments. YS, LL, HC, TP, and $X L$ carried out the experiments. All authors analyzed the data. $Y S, L L$, and $W Y$ written the manuscript. All authors read and approved the final manuscript.

\section{Funding}

This work was supported by the National Natural Science Foundation $(31401688,31772140)$ to Wenwu Ye, and the National Natural Science Foundation (31721004) to Yuanchao Wang.

\section{Availability of data and materials}

Not applicable.

Ethics approval and consent to participate

Not applicable.

\section{Consent for publication}

Not applicable.

\section{Competing interests}

The authors declare that they have no conflict of interest.

\section{Author details}

'Department of Plant Pathology, Nanjing Agricultural University, Nanjing 210095, China. ${ }^{2}$ Present address: College of Agriculture, Ludong University, Yantai 264000, China. ${ }^{3}$ The Key Laboratory of Plant Immunity, Nanjing Agricultural University, Nanjing 210095, China. ${ }^{4}$ The Key Laboratory of Integrated Management of Crop Diseases and Pests (Ministry of Education), Nanjing 210095, China.

Received: 17 August 2020 Accepted: 27 November 2020

Published online: 04 January 2021

\section{References}

Blanco FA, Judelson HS. A bZIP transcription factor from Phytophthora interacts with a protein kinase and is required for zoospore motility and plant infection. Mol Microbiol. 2005;56:638-48.

Choi J, Kim Y, Kim S, Park J, Lee YH. MoCRZ1, a gene encoding a calcineurinresponsive transcription factor, regulates fungal growth and pathogenicity of Magnaporthe oryzae. Fungal Genet Biol. 2009;46:243-54.

Erwin DC, Ribeiro OK. Phytophthora diseases worldwide. St. Paul: APS Press; 1996.

Fang Y, Tyler BM. Efficient disruption and replacement of an effector gene in the oomycete Phytophthora sojae using CRISPR/Cas9. Mol Plant Pathol. 2016;17: $127-39$.

Gamboa-Melendez H, Huerta Al, Judelson HS. bZIP transcription factors in the oomycete Phytophthora infestans with novel DNA-binding domains are involved in defense against oxidative stress. Eukaryot Cell. 2013;12:1403-12.

Guo M, Chen Y, Du Y, Dong Y, Guo W, Zhai S, et al. The bZIP transcription factor MoAP1 mediates the oxidative stress response and is critical for pathogenicity of the rice blast fungus Magnaporthe oryzae. PLoS Pathog. 2011;7:e1001302.

He Q, Shu H, Cheng P, Chen S, Wang L, Liu Y. Light-independent phosphorylation of WHITE COLLAR-1 regulates its function in the Neurospora circadian negative feedback loop. J Biol Chem. 2005;280:17526-32.

Hua C, Wang Y, Zheng X, Dou D, Zhang Z, Govers F, et al. A Phytophthora sojae $\mathrm{G}$-protein a subunit is involved in chemotaxis to soybean isoflavones. Eukaryot Cell. 2008;7:2133-40.

Huang H-D, Lee T-Y, Tzeng S-W, Horng J-T. KinasePhos: a web tool for identifying protein kinase-specific phosphorylation sites. Nucleic Acids Res. 2005;33:W226-9.

Karin M. Too many transcription factors: positive and negative interactions. New Biol. 1990;2:126-31.

Kim S, Hu J, Oh Y, Park J, Choi J, Lee YH, et al. Combining ChIP-chip and expression profiling to model the MoCRZ1 mediated circuit for $\mathrm{Ca} /$ calcineurin signaling in the rice blast fungus. PLoS Pathog. 2010;6:e1000909.

Kong $G$, Chen Y, Deng Y, Feng D, Jiang $L$, Wan $L$, et al. The basic leucine zipper transcription factor PIBZP32 associated with the oxidative stress response is critical for pathogenicity of the lychee downy blight oomycete Peronophythora litchii. mSphere. 2020;5(3):e00261-20.

Letunic I, Doerks T, Bork P. SMART: recent updates, new developments and status in 2015. Nucleic Acids Res. 2015:43:D257-60.

Li D, Zhao Z, Huang Y, Lu Z, Yao M, Hao Y, et al. PsVPS1, a dynamin-related protein, is involved in cyst germination and soybean infection of Phytophthora sojae. PLoS One. 2013;8:e58623.

Llorca CM, Potschin M, Zentgraf U. bZIPs and WRKYs: two large transcription factor families executing two different functional strategies. Front Plant Sci. 2014;5:169.

Martin FN, Blair JE, Coffey MD. A combined mitochondrial and nuclear multilocus phylogeny of the genus Phytophthora. Fungal Genet Biol. 2014;66:19-32.

McGinnis S, Madden TL. BLAST: at the core of a powerful and diverse set of sequence analysis tools. Nucleic Acids Res. 2004;32:W20-5.

Moglich A, Ayers RA, Moffat K. Structure and signaling mechanism of per-ARNTSim domains. Structure. 2009;17:1282-94.

Molina L, Kahmann R. An Ustilago maydis gene involved in $\mathrm{H}_{2} \mathrm{O}_{2}$ detoxification is required for virulence. Plant Cell. 2007;19:2293-309.

Roy S, Poidevin L, Jiang T, Judelson HS. Novel core promoter elements in the oomycete pathogen Phytophthora infestans and their influence on expression detected by genome-wide analysis. BMC Genomics. 2013;14:106.

Schultz J, Milpetz F, Bork P, Ponting CP. SMART, a simple modular architecture research tool: identification of signaling domains. Proc Natl Acad Sci U S A. 1998;95:5857-64.

Seidl MF, Wang RP, Van den Ackerveken G, Govers F, Snel B. Bioinformatic inference of specific and general transcription factor binding sites in the plant pathogen Phytophthora infestans. PLoS One. 2012;7:e51295. 
Sheng Y, Wang Y, Meijer HJG, Yang X, Hua C, Ye W, et al. The heat shock transcription factor PSHSF1 of Phytophthora sojae is required for oxidative stress tolerance and detoxifying the plant oxidative burst. Environ Microbiol. 2015:17:1351-64.

Tamura K, Dudley J, Nei M, Kumar S. MEGA4: molecular evolutionary genetics analysis (MEGA) software version 4.0. Mol Biol Evol. 2007;24:1596-9.

Thompson JD, Higgins DG, Gibson TJ. CLUSTAL W: improving the sensitivity of progressive multiple sequence alignment through sequence weighting, position-specific gap penalties and weight matrix choice. Nucleic Acids Res. 1994;22:4673-80.

Tyler BM. Phytophthora sojae: root rot pathogen of soybean and model oomycete. Mol Plant Pathol. 2007;8:1-8.

van West P, Kamoun S, van't Klooster JW, Govers F. Internuclear gene silencing in Phytophthora infestans. Mol Cell. 1999;3:339-48.

Xiang Q, Judelson HS. Myb transcription factors in the oomycete Phytophthora with novel diversified DNA-binding domains and developmental stagespecific expression. Gene. 2010;453:1-8.

Xiang Q, Judelson HS. Myb transcription factors and light regulate sporulation in the oomycete Phytophthora infestans. PLoS One. 2014;9:e92086.

Xu F, Xu S, Wiermer M, Zhang Y, Li X. The cyclin L homolog MOS12 and the MOS4 associated complex are required for the proper splicing of plant resistance genes. Plant J. 2012;70:916-28.

Ye W, Wang X, Tao K, Lu Y, Dai T, Dong S, et al. Digital gene expression profiling of the Phytophthora sojae transcriptome. Mol Plant-Microbe Interact. 2011;24: 1530-9.

Ye W, Wang Y, Dong S, Tyler BM, Wang Y. Phylogenetic and transcriptional analysis of an expanded bZIP transcription factor family in Phytophthora sojae. BMC Genomics. 2013;14:839.

Zhang M, Lu J, Tao K, Ye W, Li A, Liu X, et al. A Myb transcription factor of Phytophthora sojae, regulated by MAP kinase PSSAK1, is required for zoospore development. PLoS One. 2012;7:e40246.

Zuo Y-H, Kang Z-S, Huang L-L, Han Q-M. Cytology on infection process of soybean hypocotyls by Phytophthora sojae. Acta Phytopathol Sin. 2005;3:23541 (in Chinese).

Ready to submit your research? Choose BMC and benefit from:

- fast, convenient online submission

- thorough peer review by experienced researchers in your field

- rapid publication on acceptance

- support for research data, including large and complex data types

- gold Open Access which fosters wider collaboration and increased citations

- maximum visibility for your research: over $100 \mathrm{M}$ website views per year

At $\mathrm{BMC}$, research is always in progress.

Learn more biomedcentral.com/submissions 